\title{
A covariance matrix-based spectrum sensing technology exploiting stochastic resonance and filters
}

\author{
Jin Lu*, Ming Huang and Jingjing Yang
}

\author{
* Correspondence: lujin211636070@ \\ sina.com \\ School of Information Science \& \\ Engineering of Yunnan University, \\ Kunming, Yunnan, China
}

\begin{abstract}
Cognitive radio (CR) is designed to implement dynamical spectrum sharing and reduce the negative effect of spectrum scarcity caused by the exponential increase in the number of wireless devices. CR requires that spectrum sensing should detect licenced signals quickly and accurately and enable coexistence between primary and secondary users without interference. However, spectrum sensing with a low signalto-noise ratio (SNR) is still a challenge in CR systems. This paper proposes a novel covariance matrix-based spectrum sensing method by using stochastic resonance (SR) and filters. SR is implemented to enforce the detection signal of multiple antennas in low SNR conditions. The filters are equipped in the receiver to reduce the interference segment of noise frequency. Then, two test statistics computed by the likelihood ratio test (LRT) or the maximum eigenvalues detector (MED) are constructed by the sample covariance matrix of the processed signals. The simulation results exhibit the spectrum sensing performance of the proposed algorithms under various channel conditions, namely, additive white Gaussian noise (AWGN) and Rayleigh fading channels. The energy detector (ED) is also compared with LRT and MED. The simulation results demonstrate that SR and filter implementation can achieve a considerable improvement in spectrum sensing performance under a strong noise background.
\end{abstract}

Keywords: Spectrum sensing, Stochastic resonance, Likelihood ratio test, Filter

\section{Introduction}

Wireless mobile network services have grown rapidly and exhibited huge potentiality in the last few decades. However, a large number of mobile terminal devices occupy spectrum resources and cause spectrum scarcity in most sub-GHz frequency bands [1]. Some frequency bands of the authorized spectrum (e.g., TV band) are already fixedly and exclusively allocated. Only partial spectrum bands are utilized at special space and time domains. This has motivated many researchers to seek innovative techniques to exploit available radio spectrum holes. Cognitive radio (CR) is considered to be a promising technology to improve the efficiency of spectrum utilization and alleviate

(c) The Author(s). 2020 Open Access This article is licensed under a Creative Commons Attribution 4.0 International License, which permits use, sharing, adaptation, distribution and reproduction in any medium or format, as long as you give appropriate credit to the original author(s) and the source, provide a link to the Creative Commons licence, and indicate if changes were made. The images or other third party material in this article are included in the article's Creative Commons licence, unless indicated otherwise in a credit line to the material. If material is not included in the article's Creative Commons licence and your intended use is not permitted by statutory regulation or exceeds the permitted use, you will need to obtain permission directly from the copyright holder. To view a copy of this licence, visit http://creativecommons.org/licenses/by/4.0/. 
the spectral congestion and shortage problem [2]. CR devices can opportunistically access the available authorized frequency bands and prevent interference.

Spectrum sensing is the principal task needed to guarantee the successful implementation of CR. Spectrum sensing requires secondary user (SU) nodes capable of executing accurate and fast detection of the idle frequency bands of primary users (PUs). Then, the SUs can be allowed to access the authorized frequency bands without any harmful interference to the PUs. Many spectrum sensing techniques have been investigated [3]. With the matched filter (MF), a priori knowledge about the PU signal is required to demodulate and detect the received signal, such as the carrier frequency, modulation format, or frame type. Energy detection (ED) is a blind algorithm that does not require any prior knowledge like the noise power but instead uses only the received signal samples to perform detection. However, ED is easily affected by noise uncertainty. Cyclostationary features detection exploits the circulating frequency of the PU signal at a low signal-to-noise ratio (SNR).

The covariance-based detector (CBD) is a mature and superior sensing technology exploiting the statistical characteristic in the sample covariance matrix of the received signal [4]. The eigenvalue-based detector depends on the framework of the generalized likelihood ratio test (GLRT) [5], whose decision statistics are constructed by a covariance matrix. For example, the maximum-minimum eigenvalue (MME) detector and the energy to minimum eigenvalue (EME) detector were proposed in [6], and it was found that the maximum eigenvalue asymptotically obeyed the Tracy Widom (TW) distribution. In practical wireless communication, the rank of the covariance matrix of primary signals is usually more than one [7]. Thus, multiple primary user models have emerged, such as the arithmetic to geometric mean (AGM) detector [8], the eigenvalue-based detector with higher order moments (EHOM) [9], and the mean-to-square extreme eigenvalue (MSEE) detector [10]. AGM can handle spectrum sensing problems with sparse samples, EHOM brings about high computational complexity, and MSEE achieves lower computational complexity.

Although eigenvalue-based spectrum sensing algorithms can improve sensing quality under the SNR wall or noise uncertainty conditions [11], in the circumstance of low SNR, increasing the number of antennas is the primary method to compensate the deterioration of spectrum sensing performance. Therefore, the design cost and complexity of wireless mobile devices will be increased. Stochastic resonance (SR) is a nonlinear physical and dynamical technology for extracting weak signals from intense noise [12]. The output of SR is determined by the dynamic characteristics, that is, the noise level, SR system, and input signal. When the noise power is proper, the system will achieve a desired state, and the output signal can be enforced. In SR, noise is an assistant rather than a disturbance or harm for signal quality. The SNR and power of a weak signal can be amplified by a nonlinear SR system, which will result in the increase of signal detection ability. The SNR wall can also be alleviated with the aid of SR.

SR is extensively applied to spectrum sensing in weak signal conditions. Energy detection based on adaptive SR is proposed through adding appropriate noise or adjusting parameters [12]. The application of SR in partial polarized noise was investigated in [13]. Other SR-based detectors have also been studied, including detectors based on suprathreshold SR [14], particle swarm algorithm and tri-stable SR [15], and optimal dynamic overdamped SR [11]. 
However, the classical SR theories point out that the input signal of an SR system can only work in low frequencies and small parameters [12], which limits its potential for wireless communication applications. Therefore, frequency shifting technologies of SR are often applied to convert high frequency of the raw signal to low frequency equivalently, such as re-sampling transformation, normalized scale transformation (NST), and generalized scale transformation (GST) [16].

A filter-based detector is another sensing scheme to separate or weaken reference noise, which does not contain the PU signal. This advantage is offered by a generalized detector (GD), which considers two filters as additional linear systems before signal processing [17]. GD exploits the statistics of the mean and variance at the filters output, and its superiority has been demonstrated in MF, ED, and correlation detector [18]. GD employment in CR systems was investigated in [19].

Motivated by the aforementioned studies, this paper designed a covariance matrixbased detector that utilizes SR and filters. Multiple antenna signals are processed via the SR system, in which an NST frequency shifting scheme is exploited. The filters are equipped in the receiver to remove the high-frequency segment of noise. Then, according to the covariance matrices of the processed signals, two test statistics are constructed by the likelihood ratio test (LRT) or the maximum eigenvalues detector (MED). The simulation results are provided to compare the detection performance of the proposed detector with the conventional detector under a strong noise background.

The rest of the paper is organized as follows: The system model is introduced in Section 2. The proposed spectrum sensing methods based on SR and filters are introduced in Section 3. Simulation results and conclusions are provided in Sections 5 and 6, respectively.

Notations: Boldface letters denote vectors or matrices. $N(a, b)$ denotes a Gaussian distribution with mean $a$ and variance $b$. The superscript $(\cdot)^{T}$ denotes the transposition. The superscript (.) denotes the conjugate transposition. Furthermore, $(.)^{-1}$ denotes the inverse of a matrix, and $\operatorname{det}(\cdot)$ denotes the determinant of a matrix.

\section{System model}

Assuming that the SU nodes are equipped with an uncorrelated antenna array in the CR system, the spectrum sensing model can be divided as a conventional binary hypothesis test problem [5]:

$$
\begin{aligned}
& H_{0}: r_{i}(n)=w_{i}(n), \\
& H_{1}: r_{i}(n)=h_{i}(n) s(n)+w_{i}(n),
\end{aligned}
$$

where the hypothesis $H_{0}$ denotes making a decision that the primary users' signals are absent, and the hypothesis $H_{1}$ denotes making a decision that the primary users are present. The variable $i$ denotes the $i$-th antenna element and $i=\{1, \ldots, M\} . M$ is the number of antennas. The variable $n$ denotes $n$-th sample time instant and $n=\{0,1, \ldots$, $\left.N_{s}-1\right\}$. In which, $N_{s}$ is the length of sensing duration. The variable $w_{i}(n)$ denotes the discrete time additive Gaussian white noise (AWGN) with mean zero and covariance $\sigma_{w}^{2}$. The joint probability distribution of $\boldsymbol{w}(n)=\left[w_{1}(n), \ldots \ldots, w_{M}(n)\right]^{\mathrm{T}}$ is $\boldsymbol{w}(n) \sim \mathcal{N}\left(0, \boldsymbol{R}_{w}\right)$, where $\boldsymbol{R}_{w}=\sigma_{w}^{2} \boldsymbol{I}_{M}$, and $\boldsymbol{I}_{M}$ is the $M \times M$ identity matrix. The variable $s(n)$ is the primary signal with the average transmitted power $E_{s}$ at the special frequency band. The signalto-noise ratio (SNR) is described as $S N R=E_{s} / \sigma_{w}^{2}$ [7]. The variable $h_{i}(n)$ denotes the 
discrete time channel coefficients representing the channel fading. It is assumed that $\boldsymbol{H}$ is the channel matrix and all antenna array elements $h_{i}(n)$ are spatially independent. The channel parameters are constant during the sensing period but differ from other sensing periods. It is assumed that $\bar{s}_{i}(n)=h_{i}(n) s(n)$, which denotes the received primary signal through channel response. Meanwhile, $\bar{s}_{i}(n)$ and $w_{i}(n)$ are independent of each other. $r_{i}(n)$ denotes the discrete time received signal of a secondary user . The observed sample signal matrix $\boldsymbol{r}$ of the primary signals captured at the secondary user during the sensing time has the dimensions $M \times N_{s}$ :

$$
\boldsymbol{r}=\left[\begin{array}{ccc}
r_{1}(0) & \ldots & r_{1}\left(N_{s}-1\right) \\
\vdots & \ddots & \vdots \\
r_{M}(0) & \ldots & r_{M}\left(N_{s}-1\right)
\end{array}\right]
$$

Then, the joint distribution of the matrix $\boldsymbol{r}$ in the hypothesis $H_{1}$ can be expressed as $\boldsymbol{r} \sim N\left(O, \boldsymbol{R}_{\bar{S}}+\boldsymbol{R}_{w}\right)$, in which $\boldsymbol{R}_{\bar{S}}$ is the sample covariance matrix of $\bar{s}$ :

$$
\boldsymbol{R}_{\bar{s}}=\frac{1}{N_{s}} \sum_{n=0}^{N_{s}-1} \overline{\boldsymbol{s}}(n) \overline{\boldsymbol{s}}^{*}(n)
$$

\section{Proposed method}

This section will introduce the proposed spectrum sensing method based on SR and filters.

\subsection{Stochastic resonance}

In order to recover the periodicity of the original signal furthest from intensive noise, the received signal in each antenna $\boldsymbol{r}_{i}(i=1, \ldots, M)$ will be processed via the SR system. When the received signal is continuous, the SR output signal is defined as:

$$
\boldsymbol{x}(t)=f(\boldsymbol{r}(t))=\left[x_{1}(t), x_{2}(t), \cdots, x_{M}(t)\right]^{T}, \quad-\infty<t<\infty,
$$

where $f($.$) is a nonlinear function representing the physical behavior. The transform-$ ation process can be defined by the Langevin equation [11]:

$$
\frac{d x_{i}(t)}{d t}=-\frac{U\left(x_{i}\right)}{d x_{i}}+\bar{S}_{i}(t)+W_{i}(t)
$$

where $U\left(x_{i}\right)$ denotes the potential function, the expression and function curve are shown as follows:

$$
U\left(x_{i}\right)=-\frac{a}{2} x_{i}^{2}+\frac{b}{4} x_{i}^{4},
$$

in which $a>0$ and $b>0$. Equation (5) indicates the classic and nontrivial SR model. The dynamic and integral characteristics of SR are driven by three basic elements: the bi-stable nonlinear system, the Gaussian white noise $w_{i}(t)$, and the external excitation $\bar{s}_{i}$ $(t)$. In Fig. 1 , the curve of $U\left(x_{i}\right)$ exists three extreme points $P_{1}\left(x_{m},-U_{0}\right), P_{2}\left(-x_{m},-U_{0}\right)$, and $O(0,0)$. In which, $x_{m}=\sqrt{a / b}$ and $U_{0}=a^{2} /(4 b)$. The two minimum points $P_{1}$ and $P_{2}$ are named as potential wells. Accordingly, the maximum point $O$ is named as potential barrier. The difference between the potential barrier and the potential wells repre- 


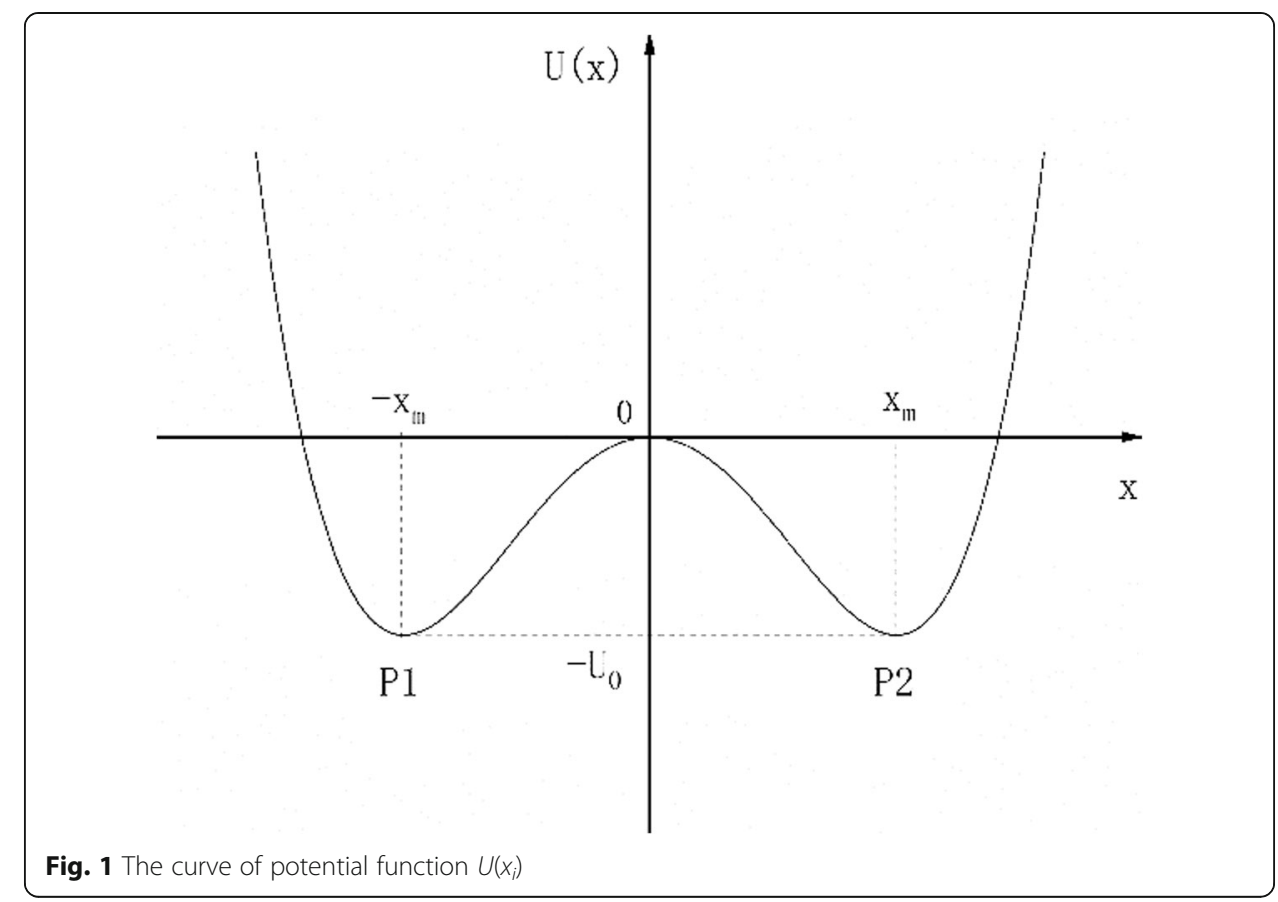

sents the potential barrier height (i.e., $U_{0}$ ). Equation (5) is a bi-stable structure because the two potential wells represent two stable states.

Supposing that a Brown particle lies in a certain point of $U\left(x_{i}\right)$ at the initial time instant, and $\bar{s}_{i}(t)$ is treated as a periodic signal with amplitude $A_{m}$ and carrier frequency $f_{c}$, there is a critical value $A_{c}=\sqrt{4 a^{3} /(27 b)}$ in $U\left(x_{i}\right)$. When the SR system is only driven by external signal $\bar{s}_{i}(t)$ and $A_{m}>A_{c}$, the particle can jump across the potential barrier, and the balance of $U\left(x_{i}\right)$ will be broken. Then, the potential wells will elevate alternatively and periodically with the frequency $f_{c}$ [14]. When only noise $w_{i}(t)$ exists, the Brown particle will switch between two potential wells with the transition speed $r_{k}$, which is named as Kramers rate [15]:

$$
r_{k}=\frac{a}{\sqrt{2} \pi} \exp \left(-\frac{2 U_{0}}{\sigma_{w}^{2}}\right)
$$

The joint effect of input $\bar{s}_{i}(t)$ and noise $w_{i}(t)$ will resonate the SR system to achieve as high an amplitude as possible. But the classical SR theory, like adiabatic approximation theory and nonlinear response theory, indicates that SR can only work with small parameters [15]. Firstly, the output signal $x_{i}(t)$ is mainly concentrated on low frequency components rather than higher harmonics, so the input carrier frequency should be a considerably small value (i.e., $f_{c} \ll 1$ and $f_{c} \ll r_{k}$ ). Secondly, the amplitude $A_{m}$ and the noise power $\sigma_{w}^{2}$ should also be far less than 1 .

The prerequisite of low frequency is a straight conflict of the modulation carrier requirement of high frequency in wireless communication. To solve this problem, we exploit the normalized scale transformation (NST) method to convert high frequency to low frequency [16]. The NST technology exploits the normalization and variable substitutions as follows: 


$$
\frac{d z_{i}(\tau)}{d t}=z_{i}-z_{i}^{3}+A_{0} \cos \left(2 \pi f_{0} \tau\right)+w_{0}(\tau)
$$

where $z=x \sqrt{a / b} ; \tau=a t$ is the re-sample time interval; $A_{0}=A_{m} \sqrt{b / a^{3}}$ is the normalized amplitude; $w_{0}(\tau)$ is the normalized noise with expectation 0 and variance $\sigma_{0}^{2}=\sigma_{w}^{2} b$ $/ a^{2}$; and $f_{0}$ is the normalized frequency and can be expressed by the original carrier frequency (i.e., $f_{0}=f_{c} / a$ ).

It can be found that Eq. (8) is the standard normalized form of Eq. (5), and they have the same dynamic characteristics. However, the main significances and contributions lie in that Eq. (8) can satisfy the preconditions of small parameters according to the adiabatic approximation theory. Note that the condition $a \gg 1$ can ensure that the high frequency $f_{c}$ of the carrier signal turns into a low frequency $f_{0}$. Thus, for algorithm implementation, we can preset small values of $f_{0}$ and $A_{0}$ to obtain $a$ and $b$. Then, $f_{0}$ and $A_{0}$ can be adjusted based on the output SNR and resonance effect to achieve the desired state.

In general, Eq. (8) is an expression of an ordinary first order differential equation, and no exact solutions have been provided in recent studies. However, it can be approximately solved by the fourth order Runge Kutta (RK) algorithm [13], which is a numerical computation method and includes the process of multi-stage iteration. Meanwhile, RK algorithm is the form of discrete time system referred to the Langevin Eq. (5), and processes $r_{i}(n)$ rather than $r_{i}(t)$.

\subsection{Filters}

The output of SR $x_{i}(n)$ will pass through two linear time invariant discrete time systems, that is, low pass, high pass, or band pass filters. The two filters are named as preliminary filter (PF) and additional filter (AF) with the impulse responses $h_{p}(m)$ and $h_{a}(m)$, respectively, which is mentioned in a description of GD in [19]. The processes of PF and AF are described as a convolution form:

$$
\left\{\begin{array}{l}
e_{i}(n)=\sum_{m=-\infty}^{\infty} h_{p}(m) x_{i}(n-m) \\
\eta_{i}(n)=\sum_{m=-\infty}^{\infty} h_{a}(m) x_{i}(n-m)
\end{array}\right.
$$

where $e_{i}(n), n=0, \ldots, N_{s}-1$ is the $i$-th antenna and $n$-th sample of the secondary data via SR at the PF output; $\eta_{i}(n)$ is the corresponding one at AF output; and $\boldsymbol{e}$ and $\boldsymbol{\eta}$ are the $M \times N_{s}$ matrix forms of $e_{i}(n)$ and $\eta_{i}(n)$.

Assuming that the central frequencies of $\mathrm{PF}$ and $\mathrm{AF}$ are detuned, the PU signal via SR cannot pass through AF and only appears in the PF output. If the detuning of central frequencies between the AF and PF achieved over four times the PU signal bandwidth, the correlation coefficient between the PF and AF output can be ignored [18]. That means the AF and PF outputs are independent. Then, the amplitude frequency characteristics of the PF and AF can be adjusted to ensure that the noise portions are equal. Hence, it is approximately considered that $\boldsymbol{e} \approx \boldsymbol{x}+\boldsymbol{\eta}$, and the noise power can be estimated by $\boldsymbol{\eta}$. 


\subsection{Detection method}

Now that the filtered signal $\boldsymbol{e}$ and $\boldsymbol{\eta}$ are obtained, this section will introduce three detection algorithms, namely, ED, LRT, and MED.

\subsubsection{ED}

The ED algorithm is a blind spectrum sensing method used when $\bar{s}_{i}(n)$ is not known to the CR user. ED employs the sum of the energy at the observed interval. The test statistic is defined as follows [11]:

$$
T_{E D}=\sum_{i=1}^{M} \sum_{n=0}^{N_{s}-1}\left|e_{i}(n)\right|^{2},
$$

\subsubsection{LRT}

Based on the Neyman Pearson (NP) criterion, when the probability of false alarm $P_{f}$ and the noise variance $\sigma_{\eta}^{2}$ is given, the LRT will maximize the detection probability. The decision statistic of LRT is determined using the following form:

$$
T_{L}=\frac{p\left(\boldsymbol{e} \mid H_{1}\right)}{p\left(\boldsymbol{e} \mid H_{0}\right)}=\prod_{n=0}^{N_{s}-1} \frac{p\left(\boldsymbol{e}(n) \mid H_{1}\right)}{p\left(\boldsymbol{e}(n) \mid H_{0}\right)},
$$

where $\boldsymbol{e}$ is the received signal vector that is the aggregation of $\boldsymbol{e}(n)$, and $p\left(\boldsymbol{e} \mid H_{1}\right)$ and $p\left(\boldsymbol{e} \mid H_{0}\right)$ denote the likelihood function under the hypotheses $H_{1}$ and $H_{0}$, respectively. The likelihood function at time instant $n$ can be presented in the following form [13]:

$$
\begin{aligned}
& p\left(\boldsymbol{e}(n) \mid H_{1}\right)=\frac{\exp \left\{-\boldsymbol{e}^{*}(n)\left(\boldsymbol{R}_{\bar{s}}+\boldsymbol{R}_{\eta}\right)^{-1} \boldsymbol{e}(n)\right\}}{\pi^{2} \operatorname{det}\left(\boldsymbol{R}_{\bar{s}}+\boldsymbol{R}_{\eta}\right)}, \\
& p\left(\boldsymbol{e}(n) \mid H_{0}\right)=\frac{\exp \left\{-\boldsymbol{e}^{*}(n) \boldsymbol{R}_{\eta}^{-1} \boldsymbol{e}(n)\right\}}{\pi^{2} \operatorname{det}\left(\boldsymbol{R}_{\eta}\right)} .
\end{aligned}
$$

Based on the character of the matrix inversion lemma, there is

$$
\boldsymbol{R}_{\eta}^{-1}-\left(\boldsymbol{R}_{\bar{s}}+\boldsymbol{R}_{\eta}\right)^{-1}=\frac{1}{\sigma_{\eta}^{2}} \boldsymbol{R}_{\bar{S}}\left(\boldsymbol{R}_{\bar{S}}+\boldsymbol{R}_{\eta}\right)^{-1} .
$$

Hence, Eq. (11) can be simplified as follows:

$$
\sigma_{\eta}^{2} \ln \left(T_{L}\left(\frac{\operatorname{det}\left(\boldsymbol{R}_{\bar{s}}+\boldsymbol{R}_{\eta}\right)}{\operatorname{det}\left(\boldsymbol{R}_{\eta}\right)}\right)^{N_{s}}\right)=\sum_{n=0}^{N_{s}-1} \boldsymbol{e}^{*}(n) \boldsymbol{R}_{\bar{s}}\left(\boldsymbol{R}_{\bar{s}}+\boldsymbol{R}_{\eta}\right)^{-1} \boldsymbol{e}(n) .
$$

Note that the left side of Eq. (15) does not contain the signal matrix $\boldsymbol{e}$ and does not relate to constructing the test statistic. In contrast, the right side of Eq. (15) does relate to constructing the test statistic and in fact is defined as a new test statistic: $T_{L R T}$ $\lessgtr \gamma_{L R T}$, where $\gamma_{L R T}$ is the detection threshold of LRT.

\subsubsection{MED}

LRT is the optimal and ideal detector based on the likelihood function, in which some parameters, such as noise variance $\sigma_{n}^{2}$ or received source signal covariance $R_{\bar{s}}$, are 
known. In most practical scenarios, they are blind. This means the probability distribution of the observations or the likelihood functions cannot be obtained. This type of problem can only be solved by GLRT, which estimates the unknown parameters by maximum likelihood estimate (MLE). The test statistics of the GLRT detector have some simple form expressed by the eigenvalue of the sample covariance matrix of the received signal. Thus, MED will be used in this section. The algorithm steps are expressed as follows [5]:

1) Calculate the sample covariance matrix of filter output signal $\boldsymbol{e}(n)$ as:

$$
\boldsymbol{R}_{e}=\frac{1}{N_{s}} \sum_{n=0}^{N_{s}-1} \boldsymbol{e}(n) \boldsymbol{e}^{*}(n)
$$

2) Calculate the eigenvalues of the sample covariance matrix $\boldsymbol{R}_{e}$ and order them as $\lambda_{1} \geq \lambda_{2} \geq \quad \geq \lambda_{M}$

3) Use the largest eigenvalues for detection:

$$
T_{M E D}=\frac{\lambda_{1}}{\sigma_{\eta}^{2}}
$$

\subsection{Algorithm summary}

The proposed algorithm is summarized in Fig. 2. Next, the computational complexity is analyzed. Due to the RK algorithm, the extra computational cost is produced in contrast to the traditional detector. Since the signals from multi antennas are independent, the receivers can calculate the SR output concurrently. Thus, the RK algorithm needs $5\left(N_{s}-1\right)$ manipulations. The computational complexity of SR is $T_{S R}=O\left(5\left(N_{s}-1\right)\right)=$ $\mathrm{O}\left(\mathrm{N}_{\mathrm{s}}\right)$, which is linear order. It indicates that the time cost is acceptable compared to detection probability. Noted that the computational complexity only reflects the gradual change of the time complexity accompany with the problem scale $N_{s}$. $T_{S R}$ cannot reflect the time frequency, i.e., the actual execution time of the algorithm.

\section{Simulation results and discussion}

\subsection{Simulation results}

In this section, the spectrum sensing performance of ED, LRT, and MED based on SR and filters will be evaluated by simulation. Figure 3 displays the effects of SR and PF in arbitrary antenna under the hypothesis $H_{1}$ without channel fading. The simulation parameters are presented follows: The input signal is a periodic sine wave $s=A_{m} \cos (2$ $\pi f_{c} t$ ) with the amplitude $A_{m}=1$ and carrier frequency $f_{c}=10 \mathrm{~Hz}$, the normalized amplitude is $A_{0}=0.5$, the normalized frequency is $f_{0}=0.01 \mathrm{~Hz}$, the signal-to-noise ratio is $S N R=-5 \mathrm{~dB}$, the sample frequency is $f_{s}=5 \mathrm{kHz}$, and the sample number is $N_{s}=1536$. 


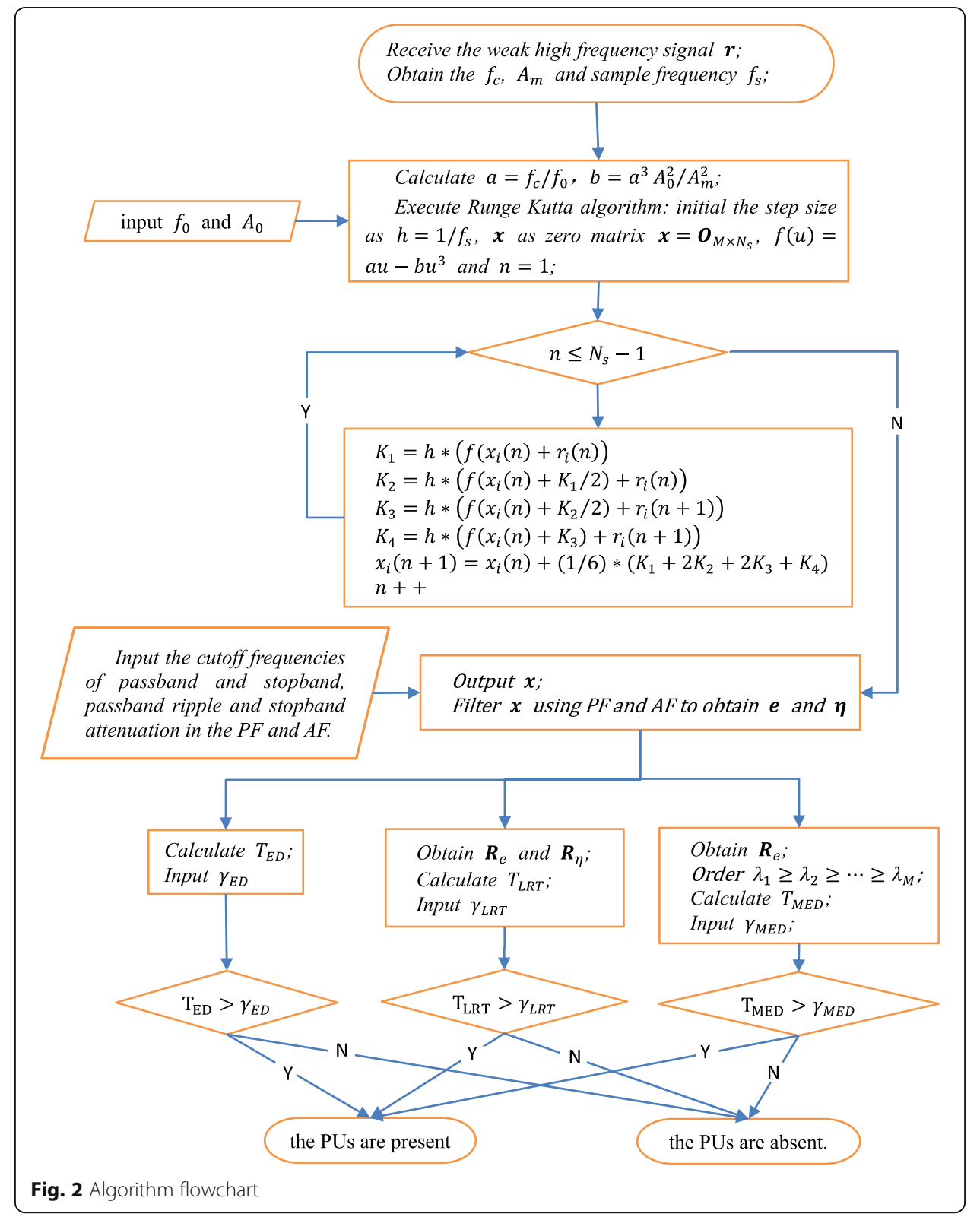

The PF is a digital low pass filter implemented by finite impulse response (FIR) based on Kaiser Window. The parameters of Kaiser Window is $\beta=4.53$. The order of FIR is 150. The cutoff frequency of pass band and stop band are $f_{p p}=100 \mathrm{~Hz}$ and $f_{p s}=200$ $\mathrm{Hz}$, respectively.

It can be found that the SR system can adequately recover the signal periodicity buried in noise. The effect of NST is obvious through adjusting the SR parameters $a$ and $b$. It appears that the SR output signal still works in the frequency $10 \mathrm{~Hz}$ rather than $0.01 \mathrm{~Hz}$. Therefore, the setting of $f_{0}$ and $A_{0}$ is rational. It is also shown that the PF can effectively reduce the noise ingredient and make the wave curve smoother.

Figure 4 shows the influence that the parameters $A_{0}$ of NST have on the detection probability $P_{d}$ under PF and AF. The parameters are presented as follows: The antenna number is $M=3$, the sample number is $N_{s}=512$, the false alarm probability is $P_{f}=0.1$, 


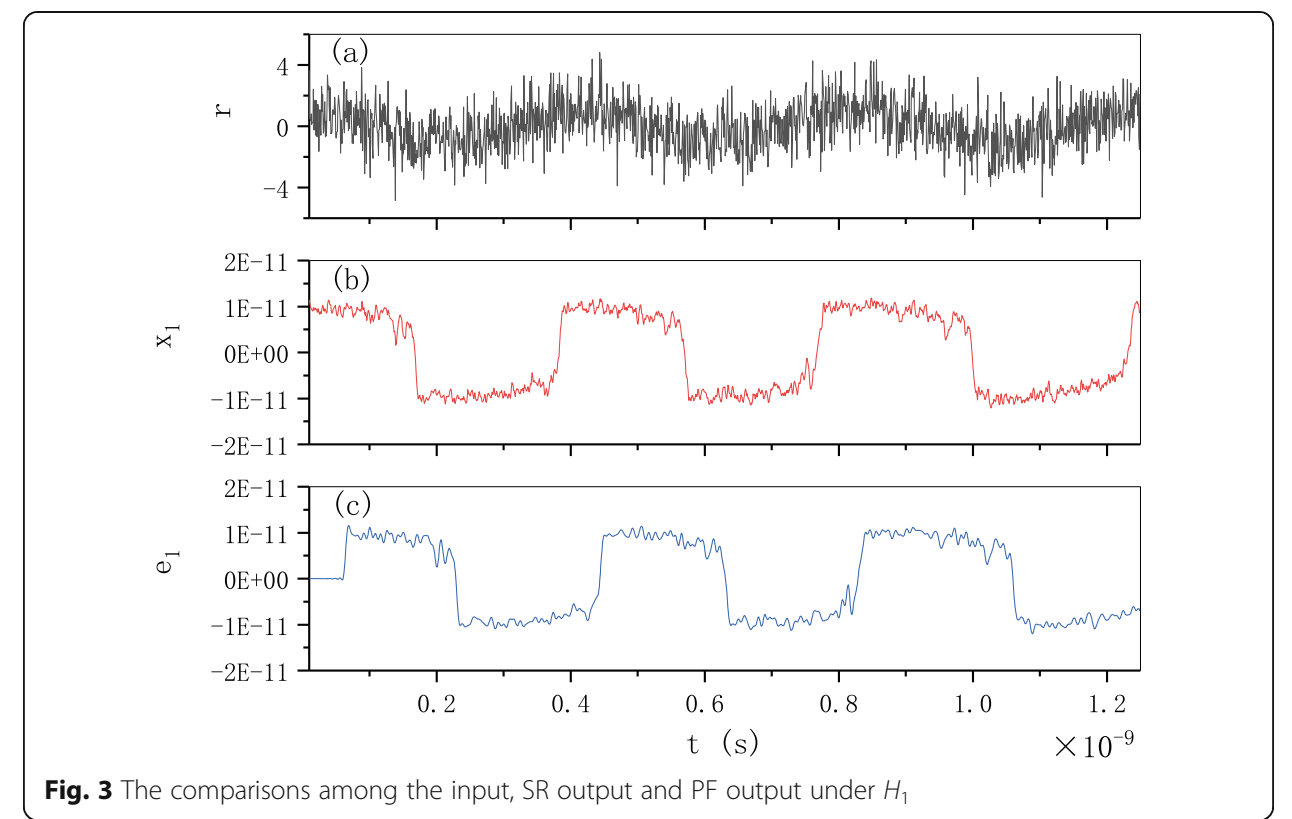

the times of Monte Carlo simulation is $5000, S N R=-16 \mathrm{~dB}, f_{p p}=500 \mathrm{~Hz}$, and $f_{p s}=600$ Hz. The AF is a high pass filter implemented by FIR based on Kaiser Window, and the cutoff frequency of stop band and pass band are $f_{a p}=1900 \mathrm{~Hz}$ and $f_{a s}=3200 \mathrm{~Hz}$, respectively. The other parameters are the same as the ones in Fig. 3. In the legends of the following figures, the abbreviations of SR-ED, SR-LRT, and SR-MED denote the SR-based detection algorithms of ED, LRT, and MED, respectively.

It is shown that, accompanied with the increase of $A_{0}$ at the interval [0.1, 0.4], $P_{d}$ promotes rapidly, and the order of detection performance from best to worst is $E D>L R T>$ $M E D$. When $A_{0}$ increases at the interval $[0.4,1], P_{d}$ descends slowly and smoothly.

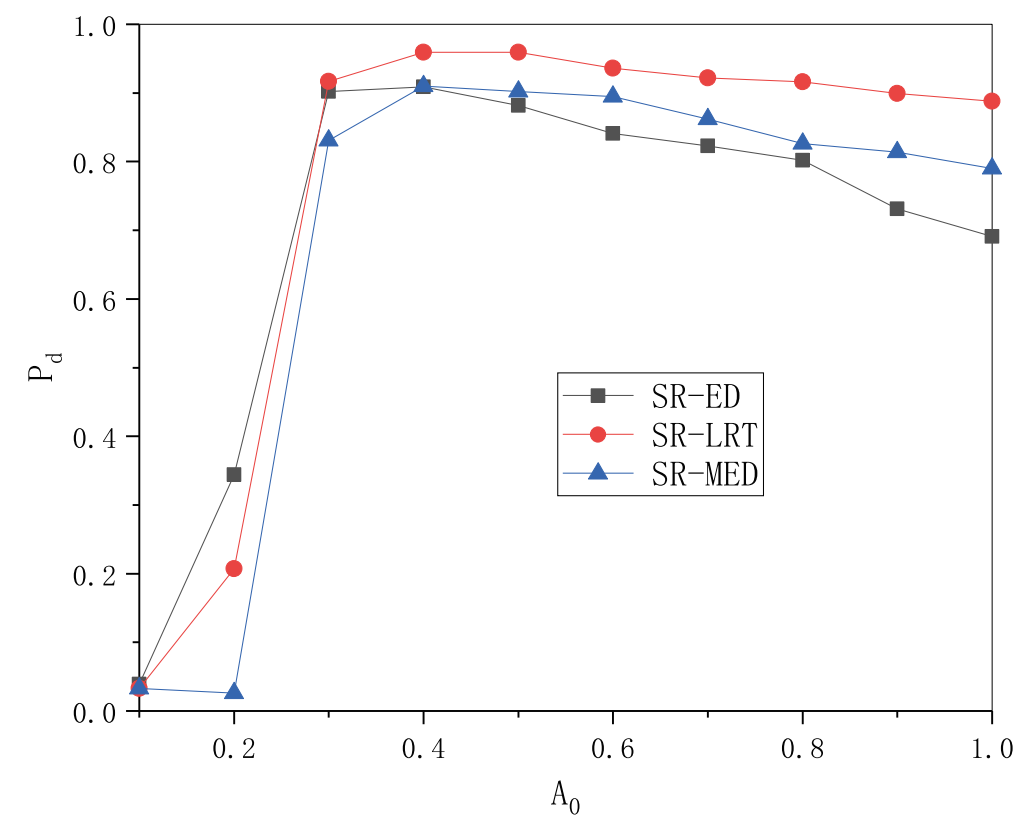

Fig. 4 The detection probability $P_{d}$ versus normalized amplitude $A_{0}$ with filters 
Meanwhile, the order of detection performance from best to worst is $L R T>M E D>E D$. The variation trends are the same for ED, LRT, and MED. It is demonstrated that the critical value of a normalized SR system is $A_{c}=0.344$ [12], which approximately coincides with the optimal value $A_{0}=0.4$ shown in Fig. 4. It is indicated that when $A_{0} \leq A_{c}$, the input signal itself cannot cross the potential barrier, while the assistant effect of noise is extremely significant until $A_{0}=A_{c}$. When $A_{0}>A_{c}$, the situation is reversed and the assistant effect of noise becomes weaker when larger $A_{0}$ values are selected.

Figure 5 tests the influence of the normalized frequency $f_{0}$ with the interval $[0.01,0.1]$ when $A_{0}=0.5$. It is shown that when $f_{0}$ is lower in value, the noise will easily satisfy the requirement of Kramers rate and produce the SR phenomena. However, considering the calculation problem of overflow, $f_{0}=0.01$ is low enough. In addition, the order of detection performance from best to worst is $L R T>E D>M E D$.

Figures 6 and 7 exhibit the variation curve of detection probability $P_{d}$ versus $S N R$ at the interval $[-24,-9] \mathrm{dB}$ without filters and with filters. The three algorithms ED, LRT, and MED based on SR are also compared. According to the simulation conclusions in Figs. 4 and 5 , the parameters are set as $f_{0}=0.01 \mathrm{~Hz}$ and $A_{0}=0.5$ while other parameters are not changed.

It is shown that SR can enormously improve the detection performance in various detection algorithms. When filters are employed, the advantage of SR is weakened compared with the situation without filters. However, PF and AF can help to enhance the function of SR overall. The order of detection performance from best to worst is $L R T>$ $M E D>E D$ whether SR and filters are employed or not.

Figures 8 and 9 consider the wireless communication circumstances of Rayleigh fading channel and exhibit the variation curve of detection probability $P_{d}$ versus $S N R$ when filters are exploited or not. ED, LRT, and MED are also compared in Figs. 8 and 9. The parameters are the same as the ones in Figs. 6 and 7 except the fading coefficient $\beta=0.5$.

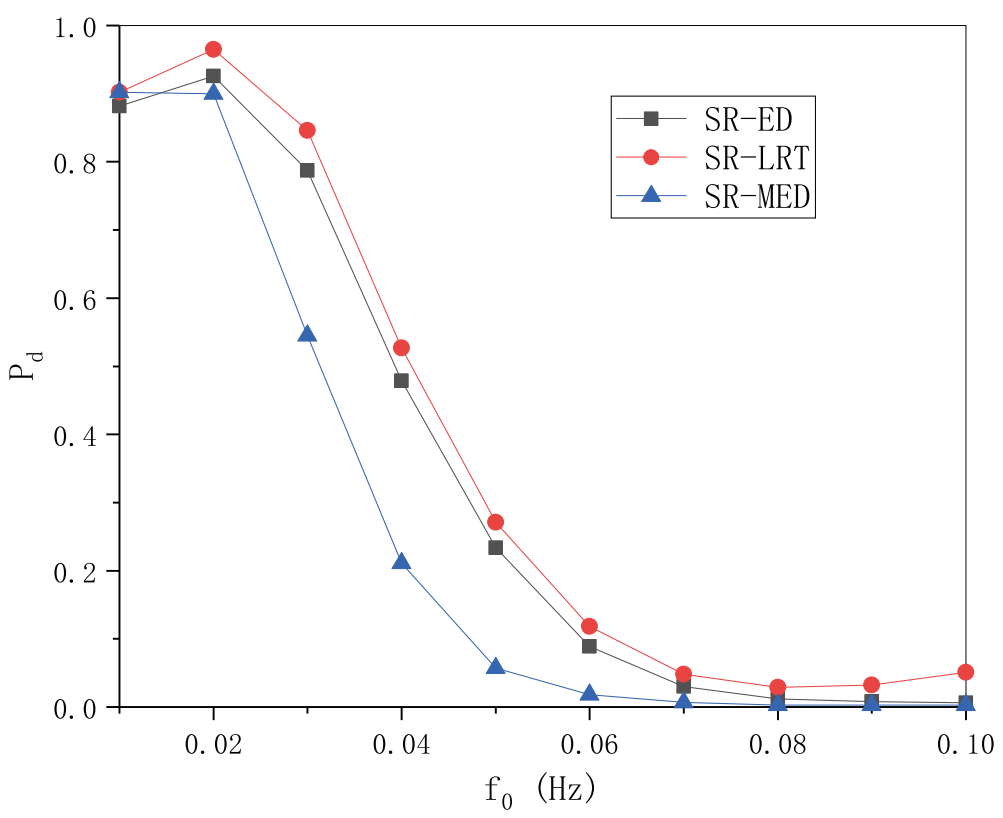

Fig. 5 The detection probability $P_{d}$ versus normalized frequency $f_{0}$ with filters 


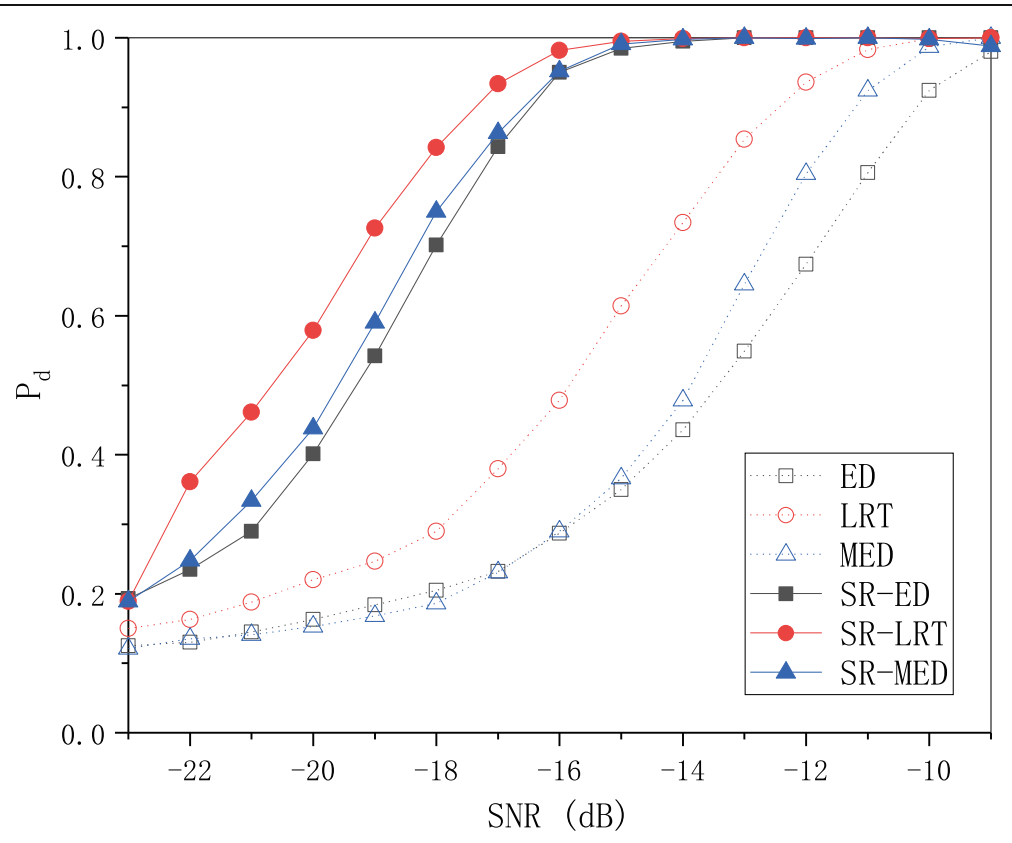

Fig. 6 The comparison of detection probability without filters

It is shown that SR can enhance the detection performance of various detection algorithms in the low SNR area at $[-23,-11] \mathrm{dB}$. In this area, the situations and effects of SR and filters are the same as in Figs. 6 and 7. However, the difference lies in that the order of detection performance from best to worst is $L R T>E D>M E D$ whether SR and filters are employed or not. This indicates that when noise power is unknown, ED is the preferred alternative scheme rather than MED.

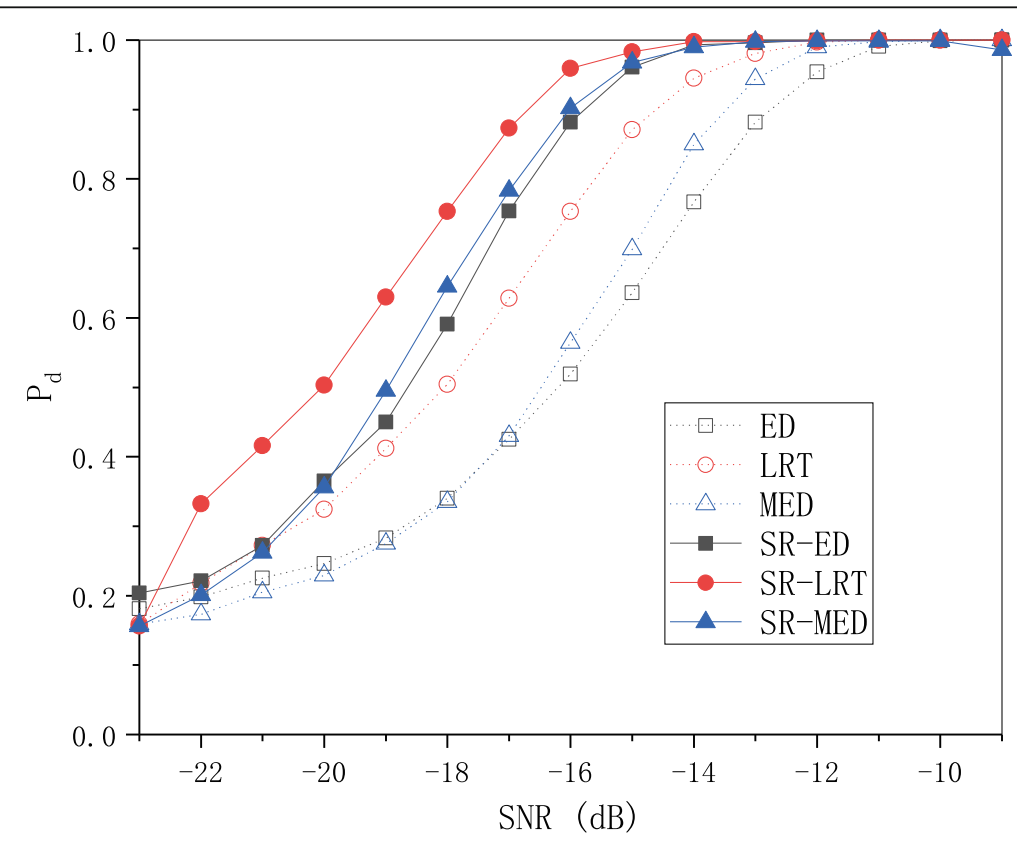

Fig. 7 The comparison of detection probability with filters 


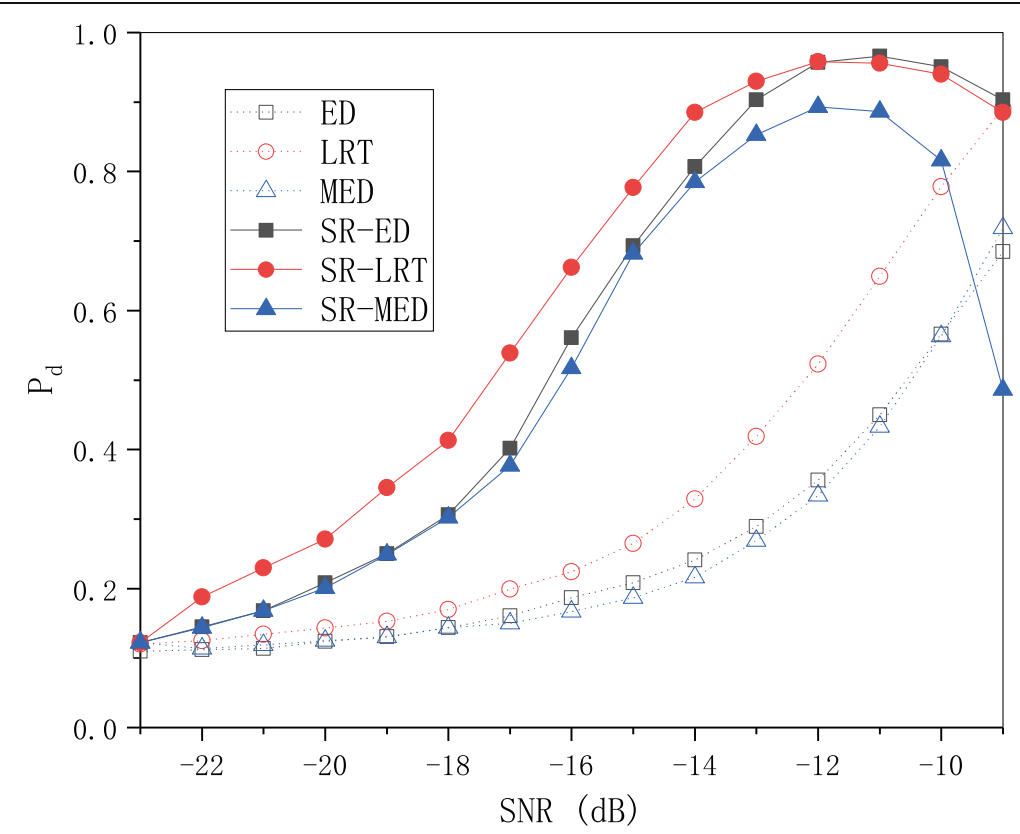

Fig. 8 The comparison of detection probability under Rayleigh channel without filters

When $S N R \geq-11 \mathrm{~dB}$, the detection performance with SR descends in Rayleigh fading whether filters are employed or not. This indicates that the channel matrix $\boldsymbol{H}$ causes the external excitation $\bar{s}(n)$ to become an aperiodic signal. The relatively weak noise cannot help $\bar{s}(n)$ to jump across the potential barrier. Therefore, SR is not suitable for high SNR conditions under Rayleigh fading channel.

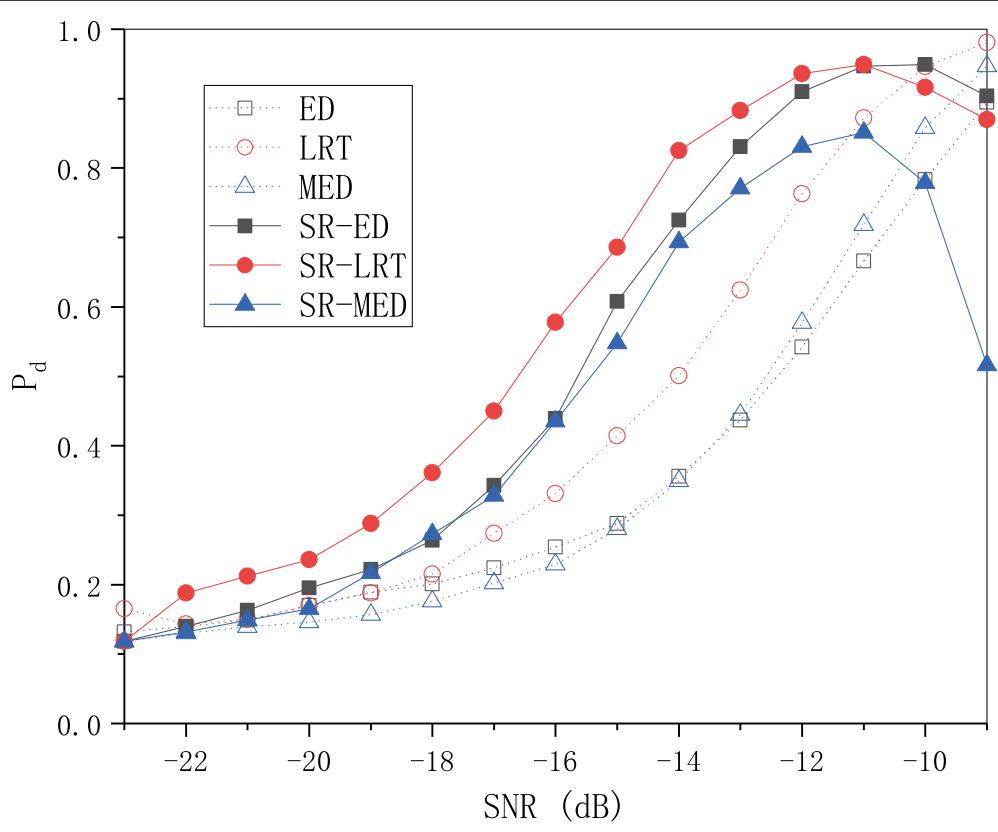

Fig. 9 The comparison of detection probability under Rayleigh channel with filters 


\section{Discussion}

From the simulation results above, it can be found that SR can enhance the output SNR under the premise of the adiabatic theory, which is ensured by the NST technology. The setting of $f_{c}=10 \mathrm{~Hz}$ in the simulation is just to demonstrate the feasibility for shifting frequency from high value to low value. When ultra-high frequency is needed, we can adjust SR parameters $a$ and $b$ to larger values.

Note that, the SR output $x_{i}(n)$ is still a high frequency signal, that is, the frequency of $x_{i}(n)$ is $f_{c}$ rather than $f_{0}$. The proposed sensing methods still work for very high frequency signals, and is applicable in various real scenarios, such as LTE system, Ad hoc, Mesh.

The distribution of SR output $x_{i}(n)$ is determined by Fokker-Planck Equation (FPE), whose exact closed-form expression is not obtained yet. Therefore, the distributions of the detection statistics $T_{E D}, T_{L R T}$ and $T_{M E D}$ cannot be analytically obtained. We have to determine the detection threshold by simulation.

To ensure that the brown particle can jump across the potential barrier, $A_{0}>0.344$ should be considered. But extremely large $A_{0}$ is harmful for detection. Additionally, smaller $f_{0}$ can easily achieve SR condition. But, we need to consider the calculation accuracy of computer device.

Under the aid of the NST technology, the filters AF and PF reduced the effect of noise component. Therefore, the filters can improve the detection performance with SR. However, the performance promotion is less obvious than the one without SR. It is probably because that the energy of SR output $x_{i}(t)$ concentrates on low frequency region, while the purpose of $\mathrm{AF}$ and $\mathrm{PF}$ is to reduce the noise in high frequency.

Additionally, SR is implemented by RK algorithm. So, more sample numbers are required to ensure the approximation accuracy. This fact will lead to the increase of execution time. In actual wireless application, we should consider the compromise of the time and accuracy.

\section{Conclusion}

This paper proposes a novel covariance matrix detector employing SR and filters. The NST technology is introduced in SR to normalized the high frequency application to a low frequency expression. The test statistic is constructed by three detector of ED, LRT or MED. The simulation results verify the extraordinary effect that SR can recover the periodicity of the received signal. It is also found that the superior detection probability is obtained when $A_{0}=0.3$ and $f_{0}=0.01 \mathrm{~Hz}$. The detection methods with SR achieve better performance than the ones without SR under Gaussian channel, while perform robust only in low SNR region under Rayleigh channel. The contributions of AF and PF is little for the detectors with SR. the test statistic of LRT performs better than the ones of ED and MED in various simulation circumstance.

\section{Abbreviations}

CR: Cognitive radio; SNR: Signal-to-noise ratio; SR: Stochastic resonance; LRT: Likelihood ratio test; MED: Maximum eigenvalues detector; AWGN: Additive white Gaussian noise; ED: Energy detector; SU: Secondary user; PU: Primary user; MF: Matched filter; CBD: Covariance based detector; GLRT: Generalized likelihood ratio test; MME: Maximum minimum eigenvalue; EME: Minimum eigenvalue; TW: Tracy Widom; AGM: Arithmetic to geometric mean; EHOM: Eigenvalue based detector with higher order moments; MSEE: Mean-to-square extreme eigenvalue; NST: Normalized scale transformation; GST: Generalized scale transformation; GD: Generalized detector; RK: Runge Kutta; PF: Preliminary filter; AF: Additional filter; NP: Neyman Pearson 


\section{Acknowledgements}

The authors would like to thank the editors and anonymous reviewers for their constructive comments and suggestions, which helped improve the manuscript. The authors also thank LetPub (www.letpub.com) for its linguistic assistance during the preparation of this manuscript. The authors are grateful to the National Science Foundation of China under Grant 61701432 for its support of this research.

\section{Authors' contributions}

The algorithms proposed in this paper have been conceived by all authors. Jin Lu designed and performed the experiments and then analyzed the results. Jin Lu wrote the paper. All authors read and approved the final manuscript.

\section{Funding}

This work is supported by the National Natural Science Foundation of China under Grant 61701432.

\section{Availability of data and materials}

Not applicable.

\section{Competing interests}

The authors declare that they have no competing interests.

Received: 18 June 2020 Accepted: 1 December 2020

Published online: 06 January 2021

\section{References}

1. G.I. Tsiropoulos, O.A. Dobre, M.H. Ahmed, K.E. Baddour, Radio resource allocation techniques for efficient spectrum access in cognitive radio networks. IEEE Commun. Surv. Tutorials. 18, 824-847 (2016). https://doi.org/10.1109/comst. 2014.2362796

2. B.B. Wang, KJ.R. Liu, Advances in cognitive radio networks: a survey. IEEE J. Selected Top. Signal. Process. 5, 5-23 (2011). https://doi.org/10.1109/jstsp.2010.2093210

3. Y.H. Zeng, Y.C. Liang, A.T. Hoang, R. Zhang, A review on spectrum sensing for cognitive radio: challenges and solutions. Eurasip J. Adv. Signal. Process (2010). https://doi.org/10.1155/2010/381465

4. Y. Zeng, Y.-C. Liang, Spectrum-sensing algorithms for cognitive radio based on statistical covariances. IEEE Trans. Vehicular Technol. 58, 1804-1815 (2009). https://doi.org/10.1109/tvt.2008.2005267

5. R. Zhang, T.J. Lim, Y.-C. Liang, Y. Zeng, Multi-antenna based spectrum sensing for cognitive radios: a GLRT approach. IEEE Trans. Commun. 58, 84-88 (2010). https://doi.org/10.1109/tcomm.2010.01.080158

6. Y. Zeng, Y.C. Liang, Eigenvalue-based spectrum sensing algorithms for cognitive radio. IEEE Trans. Commun. 57, 17841793 (2009)

7. P. Zhang, R. Qiu, GLRT-based spectrum sensing with blindly learned feature under rank-1 assumption. IEEE Trans. Commun. 61, 87-96 (2013). https://doi.org/10.1109/tcomm.2012.100912.120162

8. L. Huang, J. Fang, K. Liu, H.C. So, H. Li, An eigenvalue-moment-ratio approach to blind spectrum sensing for cognitive radio under sample-starving environment. IEEE Trans. Vehicular Technol. 64, 3465-3480 (2015). https://doi.org/10.1109/ tvt.2014.2359217

9. S. Sedighi, A. Taherpour, S. Gazor, T. Khattab, Eigenvalue-based multiple antenna spectrum sensing: higher order moments. IEEE Trans. Wirel. Commun. 16, 1168-1184 (2017). https://doi.org/10.1109/twc.2016.2640299

10. K. Bouallegue, I. Dayoub, M. Gharbi, K. Hassan, Blind spectrum sensing using extreme eigenvalues for cognitive radio networks. IEEE Commun. Lett. 22, 1386-1389 (2018). https://doi.org/10.1109/lcomm.2017.2776147

11. D. He, X. Chen, L. Pei, L.G. Jiang, W.X. Yu, Improvement of noise uncertainty and signal-to-noise ratio wall in spectrum sensing based on optimal stochastic resonance. Sensors. 19, 1-17 (2019). https://doi.org/10.3390/s19040841

12. J. Wang, X. Ren, S.W. Zhang, D.M. Zhang, H.S. Li, S.Q. Li, Adaptive bistable stochastic resonance aided spectrum sensing. IEEE Trans. Wirel. Commun. 13, 4014-4024 (2014). https://doi.org/10.1109/twc.2014.2317779

13. J. Lu, M. Huang, J. Yang, Study of polarization spectrum sensing based on stochastic resonance in partial polarized noise. Wirel. Netw 25, 4991-4999 (2019)

14. Q.W. Li, Z. Li, A novel sequential spectrum sensing method in cognitive radio using suprathreshold stochastic resonance. IEEE Trans. Vehicular Technol. 63, 1717-1725 (2014). https://doi.org/10.1109/tvt.2013.2287616

15. J. Lu, M. Huang, J.J. Yang, A novel spectrum sensing method based on tri-stable stochastic resonance and quantum particle swarm optimization. Wirel. Pers. Commun. 95, 1-13 (2017)

16. D. Huang, J. Yang, J. Zhang, H. Liu, An improved adaptive stochastic resonance with general scale transformation to extract high-frequency characteristics in strong noise. Int. J. Mod. Phys. B. 32, 185-205 (2018)

17. V. Tuzlukov. Generalized approach to signal processing in wireless communications: the main aspects and some examples. (InTech, 2012).

18. M.S. Shbat, V. Tuzlukov, SNR wall effect alleviation by generalized detector employed in cognitive radio networks. Sensors. 15, 16105-16135 (2015). https://doi.org/10.3390/s150716105

19. M. Shbat, V. Tuzlukov, Primary signal detection algorithms for spectrum sensing at low SNR over fading channels in cognitive radio. Digit. Signal Process. 93, 187-207 (2019). https://doi.org/10.1016/j.dsp.2019.07.016

\section{Publisher's Note}

Springer Nature remains neutral with regard to jurisdictional claims in published maps and institutional affiliations. 